\title{
Evaluation of the effective porosity of an open cell foam material for using in heat and mass transfer numerical simulations
}

\author{
Olga Soloveva ${ }^{1}$, Sergei Solovev ${ }^{1,}{ }^{*}$, Rimma Zaripova ${ }^{1}$, Faniia Khamidullina $^{2}$, and Marina \\ Tyurina $^{3}$ \\ ${ }^{1}$ Kazan State Power Engineering University, 51, Krasnoselskaja str., 420066, Kazan, Russia \\ ${ }^{2}$ Kazan Innovative University named after V.G. Timiryasov, 42, Moskovskaja str., 420111, Kazan, \\ Russia \\ ${ }^{3}$ Kazan National Research Technical University named after A.N. Tupolev-KAI, 10, K.Marx St., \\ 420111, Kazan, Russia
}

\begin{abstract}
A comparison of the efficiency of the open cell foam (OCF) models is carried out. Geometrical models are constructed by the methods of simple cubic, body-centered cubic, and face-centered cubic. The parameter of the material efficiency is defined as the composition of the material surface area and the gas residence time in the porous media. The analytical evaluation shows different values of effective porosity for different construction models of the material. In this case, for all considered models, the effective porosity is in the range of $0.741-0.821$.
\end{abstract}

\section{Introduction}

The open cell foam material is used in many industries. Low aerodynamic resistance, large surface area, and disordered cells' arrangement make it possible to use them as aerosol filters $[1,2]$. As a rule, filters based on OCF are used in contrast to granular filters with spherical elements [3], the porosity of which is limited by the limit of granule packing. In turn, the use of granular filters can be expanded through porous granules [4].

The disordered geometrical structure and the possibility of mixing flows inside the porous medium make it possible to use OCF as a heat exchanger [5-8]. Simultaneously, the porous structure's continuity makes it possible to remove or supply heat in a gaseous medium effectively. At the same time, the large surface area in combination with low resistance makes it possible to use OCF as catalysts for processes with wall surface reactions [9-13]. In [14-18], the use of wireless technologies in the field of automation of technological processes is considered, which can also be used to study heat transfer in OCF materials.

In this work, ordered OCF models are built using simple cubic (SC), body-centered cubic (BCC), and face-centered cubic (FCC) methods. The models are built in a wide range of porosity values. For the constructed models, the following were investigated: porosity, the surface area of solid material, gas residence time in a porous medium.

* Corresponding author: solovev.sa@kgeu.ru 
Since real porous materials are structurally a complex stochastic medium, various studies resort to various methods for creating porous structures, which can be divided into two categories - these are models with the ordered and disordered (random) arrangement of pores in space. One of the first methods for modeling porous structures is the application of the lattice Boltzmann method. The advantage of this method is that it is not demanding on computing power and at the same time gives very accurate results $[19,20]$. In cases where the structure's high porosity is not required, the method of bulk granules can be used to simulate porous structures [21, 22]. Studies of this method have shown that it gives the most accurate results at low Reynolds numbers, at low and medium porosity [23]. The next method involves the use of a Kelvin unit cell, which is a tetrakaidecahedron consisting of 14 faces, 8 regular hexagons, and 6 squares, this model gives good results in a laminar flow regime; however, in real porous structures, the flow often corresponds to a turbulent regime, which makes it difficult to use the method in the study of porous structures in close to real conditions [24]. In [25], the unit cell model was used, which is based on the Veer Phelan structure, which is an alternative to the Kelvin cell. A distinctive feature of this unit cell model is that it structurally consists of two types of cells, an irregular dodecahedron and a tetrakaidecahedron with two hexagonal and twelve pentagonal faces. The authors of [26] investigated 3 modeling methods under the assumption that the cell is a sphere, they compared models of different arrangement of spheres in space, these are bcc (bodycentered cubic unit cell) and fcc (face-centered unit cell). These models are references to the crystal lattices of metals and alkalis, there is also the sc method (primitive cubic unit cell). The sc method assumes the location of cells at the vertices of the cube, the bcc method assumes the location of another cell in the center of the cube, in the fcc method, the cells are located at the vertices of the cube and in the centers of the faces. In [27], a comparative study of the fcc and bcc methods was carried out, the authors came to the conclusion that the pressure drop is higher in the case where the fcc method was used, and the dependence of the pressure drop on the flow rate itself is linear for both cases. The most accurate method for modeling porous structures at the moment is the method of scanning real porous structures using computed tomography, after which a three-dimensional model of the structure is created on the basis of the scanned drawings [28, 29]. The main disadvantage of this method is the impossibility of adjusting the porous structure's geometric parameters, such as the diameter of the cells, linear and volumetric porosity. Attempts are also being made to create porous structures with a random arrangement of cells in space, which would be the closest in structural features to real porous materials [30, 32].

\section{Problem formulation and solution method}

\subsection{Open cell foam model}

To build an OCF material geometry is often used inverse to the packing of intersecting spheres. In turn, the packing of spheres can be constructed in different ways, for example, by randomly filling the spheres into a domain and setting the possibility of their intersection. In such case, it is not easy to pre-determine the porosity of the final geometry. Along with the random packing of spheres, there are models of ordered structures such as sc, bcc, and fcc. Examples of such structures are shown in Figure 1 (a) - (c). 


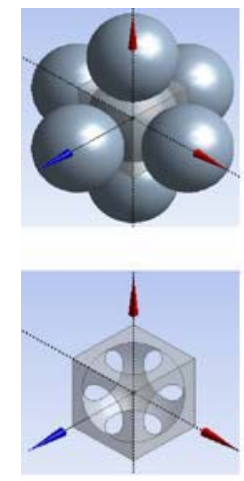

(a)
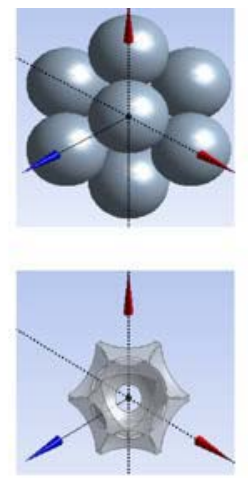

(b)

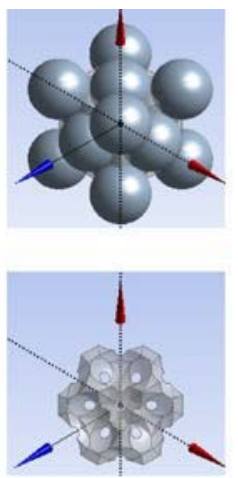

(c)

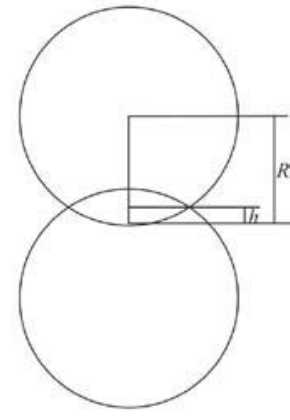

(d)

Fig. 1. Models of an OCF periodic element: (a) - sc, (b) - bcc, (c) - fcc, (d) - layout of two interpenetrating spheres

For sc, bcc, and fcc models, geometric parameters such as pore volume, surface area, and porosity are predefined. To calculate the corresponding parameters, we can use formulas [19] obtained for a cubic element.

Cubic element face length

$$
\begin{aligned}
& a_{\mathrm{sc}}=2(R-h), \\
& a_{\mathrm{bcc}}=4 \sqrt{3}(R-h) / 3, \\
& a_{\mathrm{fcc}}=2 \sqrt{2}(R-h) .
\end{aligned}
$$

Cubic element pore volume

$$
\begin{aligned}
& V_{\mathrm{sc}}=4 / 3 \pi R^{3}-2 \pi h^{2}(3 R-h), \\
& V_{\mathrm{bcc}}=8 / 3 \pi R^{3}-16 / 3 \pi h^{2}(3 R-h), \\
& V_{\mathrm{fcc}}=16 / 3 \pi R^{3}-16 \pi h^{2}(3 R-h),
\end{aligned}
$$

Porosity

$$
\begin{aligned}
& \varepsilon_{\mathrm{sc}}=V_{\mathrm{sc}} / a_{\mathrm{sc}}{ }^{3}, \\
& \varepsilon_{\mathrm{bcc}}=V_{\mathrm{bcc}} / a_{\mathrm{bcc}}{ }^{3}, \\
& \varepsilon_{\mathrm{fcc}}=V_{\mathrm{fcc}} / a_{\mathrm{fcc}}{ }^{3},
\end{aligned}
$$

Cubic element surface area

$$
\begin{aligned}
& S_{\mathrm{sc}}=4 \pi R^{2}-12 \pi h R, \\
& S_{\mathrm{bcc}}=8 \pi R^{2}-32 \pi h R, \\
& S_{\mathrm{sc}}=16 \pi R^{2}-96 \pi h R,
\end{aligned}
$$




\subsection{Evaluation of gas dynamics in porous media}

When evaluating gas dynamics, we are interested in the residence time through a porous medium (contact time). This parameter is important if an OCF material is used as a catalyst or heat exchanger. If an OCF material is used as a filter, then this parameter will also be relevant when the diffusion mechanism of particle deposition prevails.

Let us consider the gas motion with a velocity $U_{\text {in }}$ in a channel where an OCF material with a porosity $\varepsilon$ calculated in section 2.1 is located. We assume that the porosity is uniform throughout the volume of the material. Then the gas velocity movement through the porous material can be estimated as

$$
U=U_{\text {in }} / \varepsilon .
$$

Since the porosity $\varepsilon<1$, the lowest value of the velocity will be at the highest value of $\varepsilon$. A decrease in the velocity of gas movement in a porous media leads to an increase in the contact time of the gas with the surface of the porous material, which we can estimate as

$$
t=L / U
$$

where $L$ is the length of the porous region. For a unit volume of a porous region with unit length, we have $t=1 / U=\varepsilon / U_{\text {in }}$.

In addition to the contact time of the gas with the surface, the area of this surface also plays an important role. The surface area $S$ for a unit volume is defined in section 2.1. Thus, to assess the efficiency of the porous material, we will consider the value

$$
E=S t=S \varepsilon / U_{\text {in }},
$$

\section{Results}

First, let us estimate the change in the surface area of an OCF element, depending on the porosity, calculated by formulas (7) - (12), taking into account the recalculation for a cubic element of unit volume. The results are shown in Figure 2.

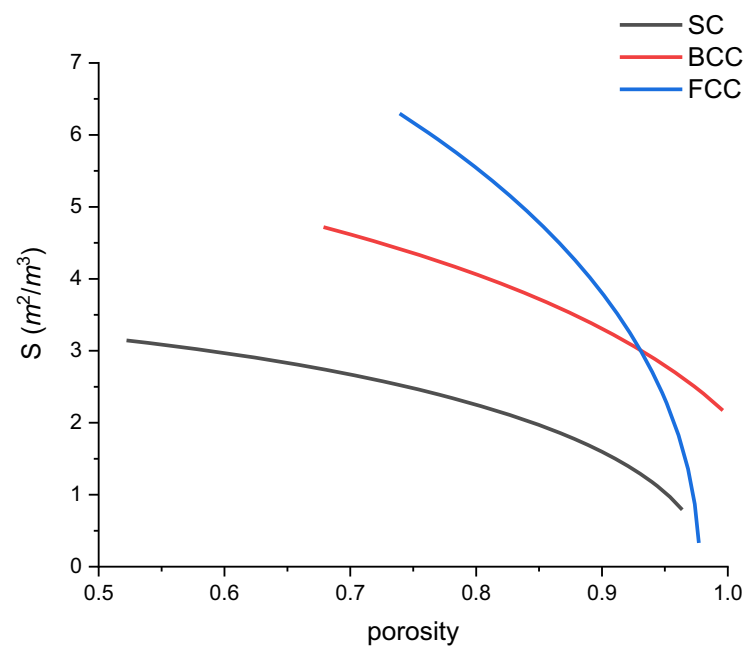

Fig. 2. Surface area of OCF element depending on the porosity. 
We see that the surface area of a porous material element decreases with increasing porosity values for all models considered (sc, bcc, and fcc). Thus, we get the largest surface area for the smallest allowable porosity value. On the other hand, a decrease in the porosity value leads to an increase in the rate of gas residence through the porous media and, accordingly, to a decrease in the time of gas contact with the material surface.

Then we will construct graphs of dependence according to the formula (15) depending on the material porosity. For simplicity, let's choose $U_{\text {in }}=1$. The results are shown in Figure 3.

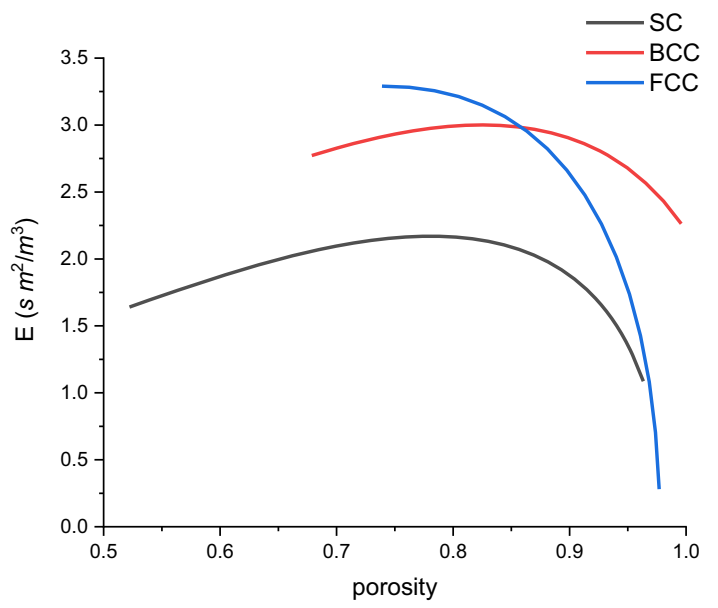

Fig. 3. Material efficiency of OCF element depending on the porosity.

Here we see that the curves have a maximum that is not on the boundary of the porosity value. In this case, the choice of a model for constructing an OCF material is essential. Thus, for the SC model, the maximum is observed at a porosity of $\varepsilon \approx 0.787$, for the BCC model $\varepsilon \approx 0.821$, and for the FCC model $\varepsilon \approx 0.741$. For the FCC model, the maximum of the introduced efficiency parameter $E$ is at the border of the permissible porosity value.

Note that, despite the differences in the calculated efficiency parameter $E$ for different geometric models of an OCF material, the effective porosity is within a small range of 0.741-0.821.

\section{Acknowledgements}

The reported research was funded by Russian Foundation for Basic Research and the government of the Republic of Tatarstan of the Russian Federation, grant № 18-41-160005.

\section{References}

1. A. Hellmann, M. Pitz, K. Schmidt, F. Haller, S. Ripperger, Aerosol Sci. Technol. 49(1), 16 (2015)

2. S.A. Solovev, O.V. Soloveva, O.S. Popkova, Rus. J. Phys. Chem. A. 92(3), 603 (2018)

3. O.V. Soloveva, S.A. Solovev, R.R. Yafizov, IOP Conf. Ser.: Mat. Sci. Eng. 709, 033064 (2020)

4. O.V. Soloveva, S.A. Solovev, R.R. Yafizov, Processes. 9(2), 268 (2021)

5. Y. Mahmoudi, N. Karimi, Int. J. Heat Mass Transf. 68, 161 (2014) 
6. M. Dehghan, M.S. Valipour, A. Keshmiri, S. Saedodin, N. Shokri, Int. J. Heat Mass Transf. 92, 815 (2016)

7. A. Hamadouche, A. Azzi, S. Abboudi, R. Nebbali, Exp. Thermal Fluid Sci. 92, 1 (2018)

8. O.V. Soloveva, S.A. Solovev, R.R. Khusainov, R.R. Yafizov, Adv. Intellig. Syst. Comp. 1259, 371 (2021)

9. L. Giani, G. Groppi, E. Tronconi, Ind. Engin. Chem. Res. 44(14), 4993 (2005)

10. S.T. Kolaczkowski, S. Awdry, T. Smith, D. Thomas, L. Torkuhl, R. Kolvenbach, Cat. Today. 273, 221 (2016)

11. S.A. Solovev, R.R. Khusainov, D.R. Nasretdinova, IOP Conf. Ser.: Mat. Sci. Eng. 618, 012096 (2019)

12. S.A. Solovev, O.V. Soloveva, I.R. Ilyasov, IOP Conf. Ser.: Mat. Sci. Eng. 734, 012205 (2020)

13. A. Kirgizov, G. Valieva, A. Laskin, I. Il'yasov, A. Lamberov, Catalysts 10(2), 250 (2020)

14. A.V. Chupaev, R.S. Zaripova, R.R. Galyamov, A.Y. Sharifullina, E3S Web Conf., 124, 03013 (2019)

15. R. Zaripova, M. Tyurina, O. Rocheva, A. Chupaev, A. Sharifullina, Adv. Econom. Business Manag. Res., 131, 271 (2019)

16. O. Rocheva, L. Khadiullina, R. Zaripova, G. Hamatgaleeva, V. Kosulin, Adv. Econom. Business Manag. Res., 131, 640 (2019)

17. R. Zaripova, A. Porunov, R.Zinurova, R. Galyamov, G. Stepanova, Adv. Econom. Business Manag. Res., 131, 663 (2019)

18. M. Tyurina, A. Porunov, R. Zaripova, A. Gaynetdinova, V. Kosulin, Adv. Econom. Business Manag. Res., 131, 648 (2019)

19. T. Heidig, T. Zeiser, H. Freund, Transp. Porous Med. 120, 207 (2017)

20. G. Falcucci, S. Ubertini, C. Biscarini, S. Di Francesco, D. Chiappini, S. Palpacelli, S. Succi, Communic. Comp. Phys. 9(2), 269 (2011)

21. G. Falcucci, S. Ubertini, D. Chiappini, S. Succi, IMA J. Applied Math. 76(5), 712 (2011)

22. K. Vafai, C. L. Tien, Int. J. Heat Mass Trans. 24(2), 195 (1981)

23. K. Vafai, J. Fluid Mech. 147, 233 (1984)

24. M. Kaviany, Princ. Heat Trans. Por. media. 119 (1995)

25. N. J. Mills, J. Mater. Sci. 40(22), 5845 (2005)

26. K. Boomsma, D. Poulikakos, Y. Ventikos, Int. J. of Heat Fluid Flow. 24(6), 825 (2003)

27. S. Krishnan, S.V. Garimella, J.Y. Murthy, J. Heat Trans. 130(2), (2008)

28. F.L. Wang, Y.L. He, S.Z. Tang, F.A. Kulacki, Y.B. Tao, Fuel. 234, 9 (2018)

29. J. Hoferer, M.J. Lehmann, E.H. Hardy, J. Meyer, G. Kasper, Chem. Engin. Techn.: Ind. Chem.-Plant Equip.-Process Engin.-Biotechn. 29(7), 816 (2006)

30. M.J. Lehmann, E.H. Hardy, J. Meyer, G. Kasper, Magn. Reson. Imaging. 23(2), 341 (2005)

31. O.V. Soloveva, S.A. Solovev, AIP Conf. Proc. 2293(1), 420034 (2020)

32. O.V. Soloveva IOP Conf. Ser.: Mat. Sci. Engin. 918(1), 012169 (2020) 